\title{
Initial Results from the PIENU experiment
}

\section{T. Sullivan ${ }^{* a}$, A. Aguilar-Arevalo ${ }^{b}$, M. Aoki $^{c}$, M. Blecher $^{d}$, D.I. Britton ${ }^{e}$, D.A. Bryman ${ }^{a}$, D. vom Bruch ${ }^{f}$, S. Chen ${ }^{g}$, J. Comfort ${ }^{h}$, S. Cuen-Rochin ${ }^{a}$, L. Doria ${ }^{i}$, P. Gumplinger $^{i}$,} A. Hussein ${ }^{j}$, Y. Igarashi ${ }^{k}$, S. Ito ${ }^{l}$, S.H. Kettell ${ }^{m}$, L. Kurchaninov ${ }^{i}$, L.S. Littenberg ${ }^{m}$, C. Malbrunot $^{n}$, R.E. Mischke ${ }^{i}$, T. Numao ${ }^{i}$, D. Protopopescu ${ }^{e}$, A. Sher ${ }^{i}$, and D. Vavilov ${ }^{i}$

${ }^{a}$ University of British Columbia, Vancouver, BC V6T 1Z1, Canada

${ }^{b}$ Instituo de Ciencias Nucleares, Universidad Nacional Autónoma de Mexico, D.F. 04510 México

${ }^{c}$ Osaka University, Toyonoka, Osaka, 560-0043, Japan

${ }^{d}$ Virginia Tech, Blacksburg, VA 24061, USA

${ }^{e}$ University of Glasgow, Glasgow, G12 8QQ, UK

${ }^{f}$ Johannes Gutenberg-University Mainz, 55122 Mainz, Germany

${ }^{g}$ Tsinghua University, Beijing, 100084, China

${ }^{h}$ Arizona State University, Tempe, AZ 85287, USA

${ }^{i}$ TRIUMF, 4004 Wesbrook Mall, Vancouver, BC V6T 2A3, Canada

${ }^{j}$ University of Northern British Columbia, Prince George, BC V2N 4Z9, Canada

${ }^{k}$ KEK, 1-1 Oho, Tsukuba-shi, Ibaraki, 305-0801, Japan

${ }^{l}$ Okayama University, Okayama, 700-0082, Japan

${ }^{m}$ Brookhaven National Laboratory, Upton, NY 11973-5000, USA

${ }^{n}$ CERN, Genéve 23 CH-1211, Switzerland

E-mail: tssullivetriumf.ca, alexis@nucleares.unam.mx,

aokimephys.sci.osaka-u.ac.jp, blechermealphamb.phys.vt.edu,

D.Britton@physics.gla.ac.uk, doug@triumf.ca,

bruch@kph.uni-mainz.de, chenshaomin@tsinghua.edu.cn,

joseph.comforteasu.edu, saulcuen@triumf.ca, luca@triumf.ca,

gumetriumf.ca, hussein@triumf.ca, youichi.igarashi@kek.jp,

s-itods.okayama-u.ac.jp, kettell@bnl.gov, kurchan@triumf.ca,

littenbedbnl.gov, chloe.mecern.ch, mischke@triumf.ca,

toshio@triumf.ca, dan.protopopesculglasgow.ac.uk, sher@triumf.ca,

vavilovetriumf.ca

The pion branching ratio, $R_{\pi}=\frac{\Gamma\left(\pi^{+} \rightarrow e^{+} v_{e}+\pi^{+} \rightarrow e^{+} v_{e} \gamma\right)}{\Gamma\left(\pi^{+} \rightarrow \mu^{+} v_{\mu}+\pi^{+} \rightarrow \mu^{+} v_{\mu} \gamma\right)}$, provides a sensitive test of lepton universality and constraints on many new physics scenarios. The theoretical uncertainty on the Standard Model prediction of $R_{\pi}$ is $0.02 \%$, a factor of twenty smaller than the experimental uncertainty. The analysis of a subset of data taken by the PIENU experiment will be presented. The result, $R_{\pi}=(1.2344 \pm 0.0023$ (stat) \pm 0.0019 (syst) $) \cdot 10^{-4}$ [1], is consistent with the Standard Model prediction and represents a $0.1 \%$ constraint on lepton non-universality.

XIII International Conference on Heavy Quarks and Leptons

22-27 May, 2016

Blacksburg, Virginia, USA

${ }^{*}$ Speaker. 


\section{Introduction}

Precision tests of the Standard Model (SM) provide a complementary approach to high-energy experiments in the search for new physics, as they can often provide tight constraints on specific extensions of the SM [2]. The $\pi \rightarrow e v$ branching ratio has been calculated within the SM to the $10^{-4}$ level; the latest theoretical prediction is $R_{\pi}=(1.2352 \pm 0.0002) \cdot 10^{-4}$ [3] [4]. The most recent experimental values, $R_{\pi}=(1.2265 \pm 0.0034$ (stat) \pm 0.0044 (syst) $) \cdot 10^{-4}$ [5] and $R_{\pi}=$ $(1.2346 \pm 0.0035$ (stat) \pm 0.0036 (syst) $) \cdot 10^{-4}$ [6], are much less precise, yet still constrain e- $\mu$ nonuniversality at the $0.2 \%$ level in charged-current weak interactions [7].

The PIENU experiment at TRIUMF aims to measure $R_{\pi}$ to a precision of less than $0.1 \%$. Presented here is an analysis of a data set collected in November 2010, containing slightly more than $4 \cdot 10^{5} \pi \rightarrow e v$ events after all selection cuts. The basic strategy of the experiment was to stop pions in an active plastic scintillator target and let them decay at rest. Almost always, the pion would then decay into a muon, which would also stop and decay via $\mu^{+} \rightarrow e^{+} v_{e} \bar{v}_{\mu}$ within the pion stopping target. The positrons produced via this process were then distinguished from the rare $\pi^{+} \rightarrow e^{+} v_{e}$ positrons by their energy and timing. The resulting statistical error was about $0.2 \%$; many systematic effects were carefully studied, and the uncertainty was ultimately reduced to slightly below the statistical error.

\section{Detector}

The PIENU detector [8] sat at the end of the TRIUMF M13 beamline, which provided a $75 \pm 0.5$ $\mathrm{MeV} / \mathrm{c}$ pion beam [9]. A cross-sectional view of the top half of the detector is shown in Figure 1. Initial beam tracking was provided by wire chambers WC1 and WC2 and silicon strip detectors $\mathrm{S} 1$ and S2; triggering and beam timing information came from the first three plastic scintillators, B1, $\mathrm{B} 2$, and $\mathrm{B} 3$, the pion stopping target.

Only events where the pion decayed at rest were included in the branching ratio calculation. The range of the $4.1 \mathrm{MeV}$ muon arising from the $\pi^{+} \rightarrow \mu^{+} v_{\mu}$ decay was insufficient to escape the target, so regardless of the decay mode undergone by the pion, a positron would emerge from the target. Only events where it entered the detector downstream of the target (relative to the beam direction) were recorded. This part of the detector consisted of another silicon strip detector S3, two more plastic scintillators $\mathrm{T} 1$ and $\mathrm{T} 2$, another wire chamber $\mathrm{WC} 3$, and finally the crystal array. WC3 and T2 were positioned very close to the front face of BINA, a large NaI(Tl) crystal that acted as an absorbtion calorimeter. BINA was surrounded by 97 smaller pure CsI crystals to capture electromagnetic shower leakage.

The positron track was obtained from S3 and WC3 and events outside a certain distance from the centre of WC3 were rejected in the analysis. This cut was nominally set at $60 \mathrm{~mm}$; the stability of the branching ratio as this parameter was varied was an important systematic check. 


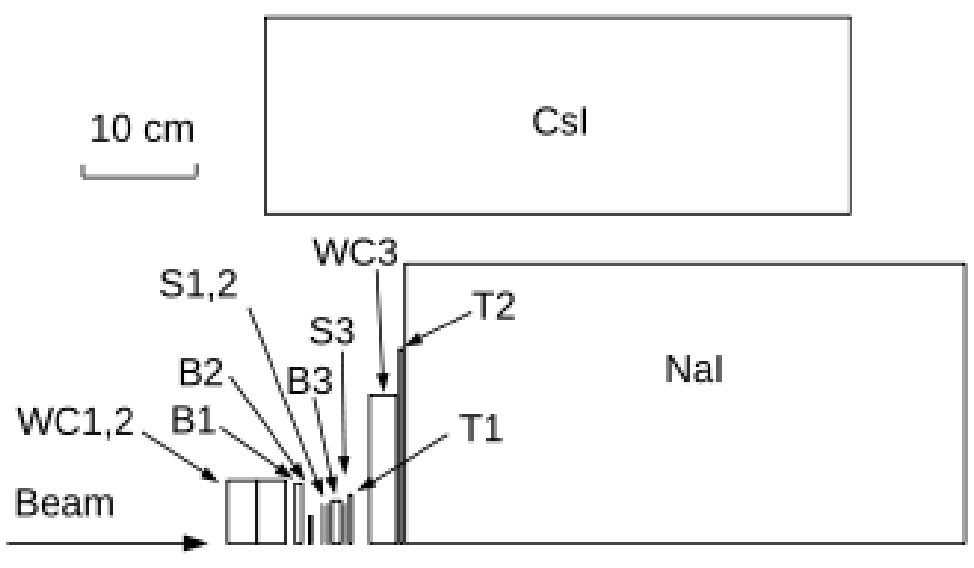

Figure 1: The PIENU Detector. See [8].

\section{Time Spectrum Analysis}

The branching ratio was obtained by dividing the events into high- and low-energy regions, according to an energy threshold $E_{\text {cut }}$, and simultaneously fitting the time spectra in the two regions. $E_{\text {cut }}$ was typically set at $52 \mathrm{MeV}$. The pion decay is a two-body process, so the total energy of the decay positron is half the pion mass, $69.8 \mathrm{MeV}$. The muon decay, by comparison, is a three-body process, and the total energy of the decay positron follows the Michel distribution, between 0.5 and $52.8 \mathrm{MeV}$. Also, the pion lifetime is much shorter than the muon lifetime: $\tau_{\pi}=26.0 \mathrm{~ns}$, whereas $\tau_{\mu}=2.197 \mu \mathrm{s}$. The time of the event was obtained by fitting the waveforms in the T1 and B1 counters, giving the times of the positron and pion hits. The difference was taken as the decay time.

The time spectra, along with all the fit components, are shown in Figure 2. The component labelled L1 is the $\pi \rightarrow \mu \rightarrow e$ signal, L2 describes pion decay-in-flight in the target, and L3 comes from events where a muon was present in the target prior to the pion, from a previous pion decay.

The high-energy spectrum is more complex. H1 is the $\pi \rightarrow e$ signal and H4 is due to a $\pi \rightarrow \mu \gamma$ decay followed by a $\mu \rightarrow e$ decay; the rest are various pileup mechanisms. H2 and H3 are the same functions as L1 and L3, respectively, but promoted to the high-energy spectrum via energy resolution or time-independent pileup. H5 and H6 describe events where an additional muon was present in the target prior to the pion, and both the "old muon" and the muon from the primary pion decayed within the integration window of the calorimeter. The two components represent different event topologies which could pass the event selection cuts. Events with multiple hits in the T1 counter were excluded in the analysis. However, some solid angle existed for a decay positron to enter the calorimeter without passing through T1. H5 represents this type of events. Furthermore, if both positrons passed through T1 sufficiently close together in time, within about $15 \mathrm{~ns}$, the two hits could not be resolved, and only one hit was recorded. H6 represents this type of events. Due to the similarity in shape between $\mathrm{H} 5$ and H6, the amplitudes were highly correlated. Rather than 


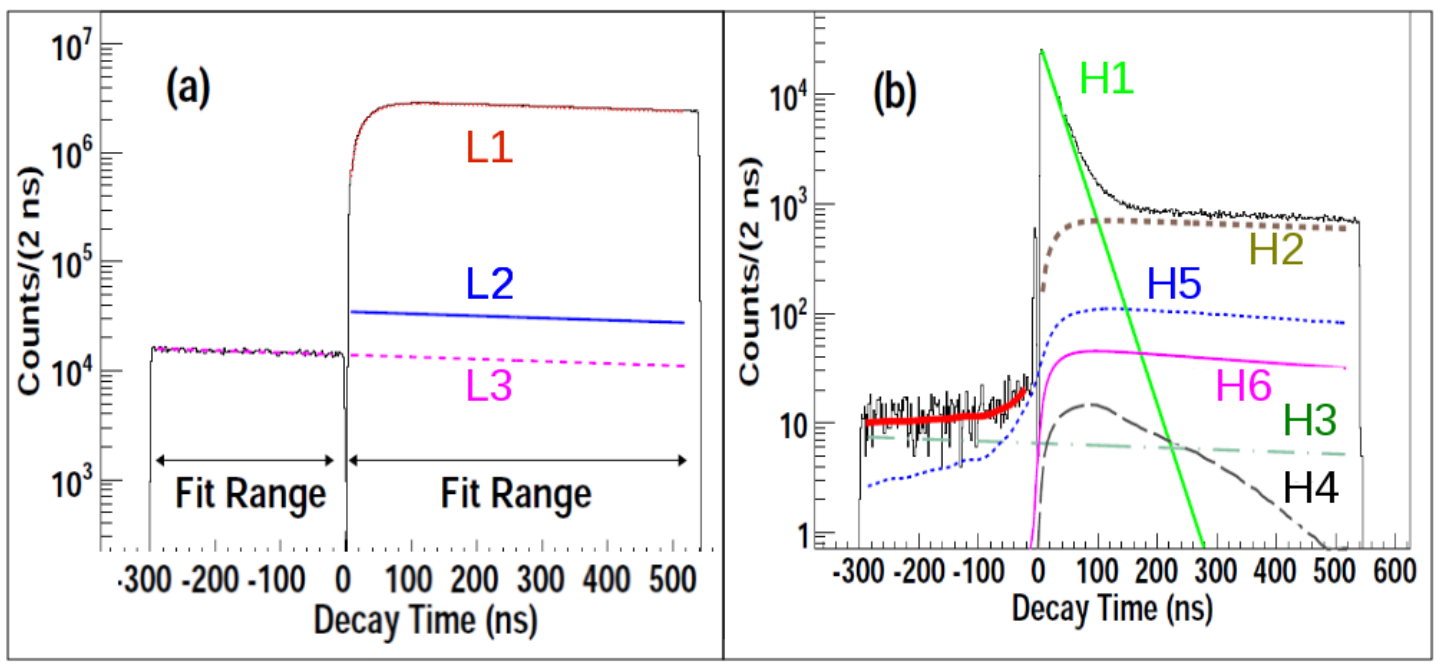

Figure 2: Time spectra of positrons in (a) the low-energy and (b) the high-energy region. The red curve in the high-energy $\mathrm{t}<0$ region is the sum of the two fit components in that region; the green curve, $\mathrm{H} 1$, in the high-energy $\mathrm{t}>0$ region is the $\pi \rightarrow e$ component; the black curve, $\mathrm{H} 4$, is due to the radiative $\pi \rightarrow \mu \gamma$ decay; $\mathrm{H} 2$ and $\mathrm{H} 3$ are $\pi \rightarrow \mu \rightarrow e$ events with neutral pileup; and H5 and H6 are $\pi \rightarrow \mu \rightarrow e$ with muon pileup (see text).

fitting both simultaneously, the shape of the former was obtained from Monte Carlo simulation and the amplitude freed in the fit, whereas the amplitude of the latter was obtained by artificially enhancing this component in the fit and fixing its ratio to L3, the pure "old muon" pileup term.

A blinding factor was introduced during the analysis process to alter the ratio of $\pi \rightarrow e$ and $\pi \rightarrow \mu \rightarrow e$ events by an unknown random factor, uniformly distributed between $\pm 1 \%$, prior to fitting. It was removed once the event selection cuts and fitting functions were finalized, and all systematic errors assigned.

Fitting these components gave a reduced $\chi^{2}$ of 1.02 with 673 DOF. The result for the raw branching ratio, the ratio of the amplitude of the $\mathrm{H} 1$ component to the amplitude of the $\mathrm{L} 1$ component, was $R_{\pi}=(1.1972 \pm 0.0022$ (stat) \pm 0.0005 (syst) $) \cdot 10^{-4}$ (after unblinding). The senstivity of $R_{\pi}$ to the fitting functions and parameters was evaluated; the systematic errors are shown in Table 1.

\begin{tabular}{c|c} 
Test & Systematic error $\left(10^{-4}\right)$ \\
\hline$\pi, \mu$ lifetimes & 0.0001 \\
Other parameters & 0.0003 \\
Excluded components & 0.0005
\end{tabular}

Table 1: Systematic errors of the raw branching ratio.

The pion and muon lifetimes were varied within their uncertainties, and also were freed in the fit; the branching ratio was stable and the lifetimes were consistent with their accepted values. The fixed amplitudes of $\mathrm{H} 4$ and H6 were varied, and a flat background was added to the fit. An 
exponential with half the muon lifetime was added as well; this type of distribution resulted in general from two muons being present in the detector simultaneously.

\section{Corrections}

The largest correction to the raw branching ratio came from the low-energy tail of the measured $\pi \rightarrow e$ energy spectrum, which caused the measured energy in approximately $3 \%$ of such events to fall below $E_{c u t}$. This component was too small to fit, so these events were lost to the branching ratio calculation; a correction therefore had to be applied.

Two independent approaches to obtaining the tail correction were used. The first used the measured energy spectrum itself, and attempted to suppress the background Michel events to a sufficient level that the low-energy tail of the $\pi \rightarrow e$ events could be extracted. The energy deposited in the calorimeter, in the time range 5-35 ns after the pion stop, is shown in Figure 3, with a line at 52 $\mathrm{MeV}$, the usual value of $E_{\text {cut }}$. Also shown is the measured energy with the additional suppression cuts applied. The $\pi \rightarrow e$ tail is estimated by subtracting the known shape of the Michel background.

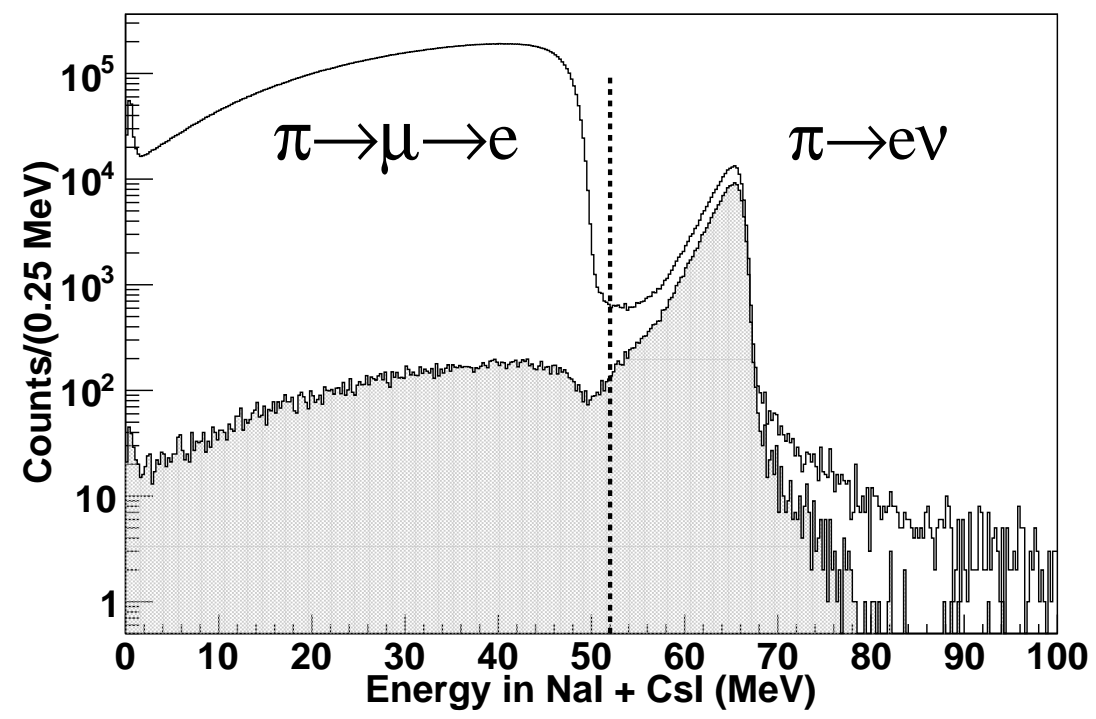

Figure 3: Measured energy spectra of positrons emerging 5-35 ns after the pion stop. The line represents the value of $E_{\text {cut }}$, and the shaded distribution has additional cuts applied to suppress $\pi \rightarrow \mu \rightarrow e$ events.

The second method for obtaining the tail correction relied on injecting a $70 \mathrm{MeV}$ positron beam into the crystal calorimeter at several angles to obtain the response function, then combining this with a Monte Carlo simulation of interactions in the plastic scintillators to get the full $\pi \rightarrow e$ tail. Positron beam data were taken at 10 angles between the crystal axis and the beam; the highest angle was 47.7 degrees. A simulation was then performed of the same data-taking configuration, and the difference between the data and simulation was used to correct the simulated $\pi \rightarrow e$ tail. 
The first method was regarded as giving a lower limit on the tail correction; $\pi \rightarrow \mu \rightarrow e$ events could not be entirely suppressed in the measured energy spectrum, particularly events where either the pion or the muon decayed in flight, and the procedure for subtracting them to obtain the $\pi \rightarrow e$ tail assumed the tail was zero at low energies, resulting in a slight oversubtraction. The second method, on the other hand, was regarded as giving an upper limit on the tail correction, since some contribution from positron scattering in the beamline could not be ruled out. The results of these two methods were combined to give a multiplicative correction of $1.0316 \pm 0.0012$ on the raw branching ratio.

Three other small corrections were applied to take into account the effect of energy-dependent scattering on the acceptance for the two decay modes, energy-dependence of the measured decay time, and muon decay-in-flight in the target, but each of these corrections was more than an order of magnitude smaller than the tail correction. To verify the corrections and fitting procedure, the stability of the corrected branching ratio against the variation of three experimental parameters was checked: the time window used to reject events with extra hits, $E_{c u t}$, and the maximum allowed distance between the reconstructed positron track and the centre of WC3. No significant dependence of $R_{\pi}$ on these parameters was observed. The variation of the branching ratio with $E_{c u t}$ is shown in Figure 4 . The branching ratio for $10 \mathrm{~mm}$ rings in the distance between the positron track and the centre of WC3 is shown in Figure 5.

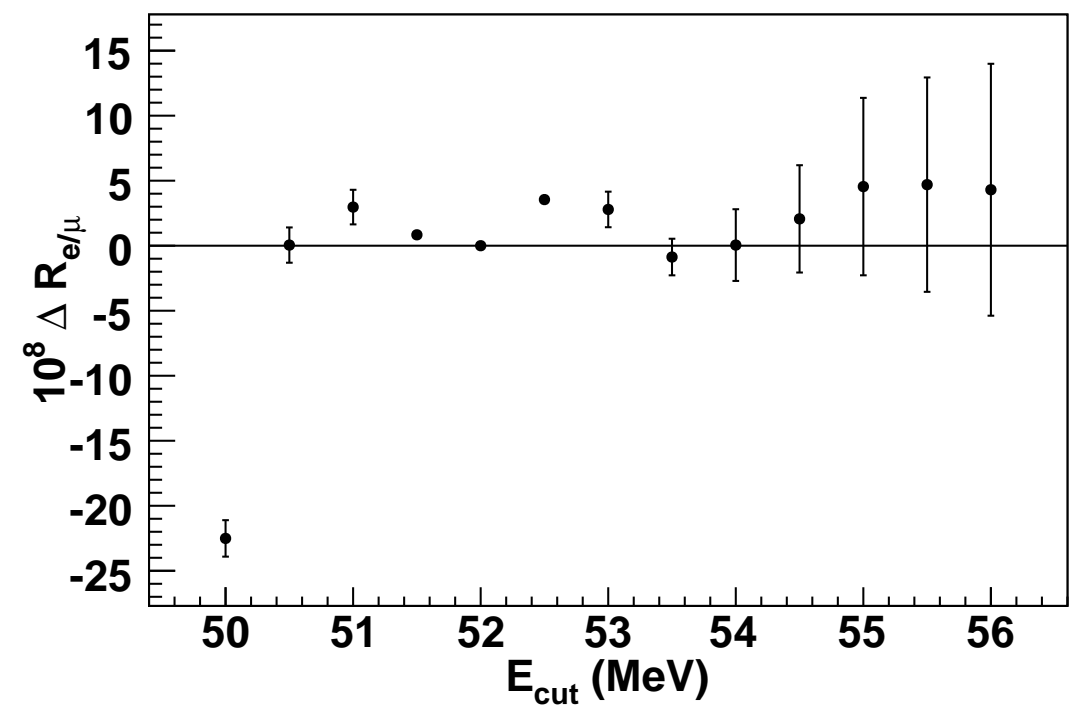

Figure 4: The change in the branching ratio as the cutoff between the low- and high-energy time spectra is varied. The error bars represent the uncertainty, both statistical and systematic, on the change from the value for the nominal cutoff $(52 \mathrm{MeV})$. Above $52 \mathrm{MeV}$, the uncertainty is largely due to the systematic error on the tail correction, and thus the error bars are correlated. The drop below $50.5 \mathrm{MeV}$ is primarily caused by the energy threshold of the trigger used for high-energy events. 


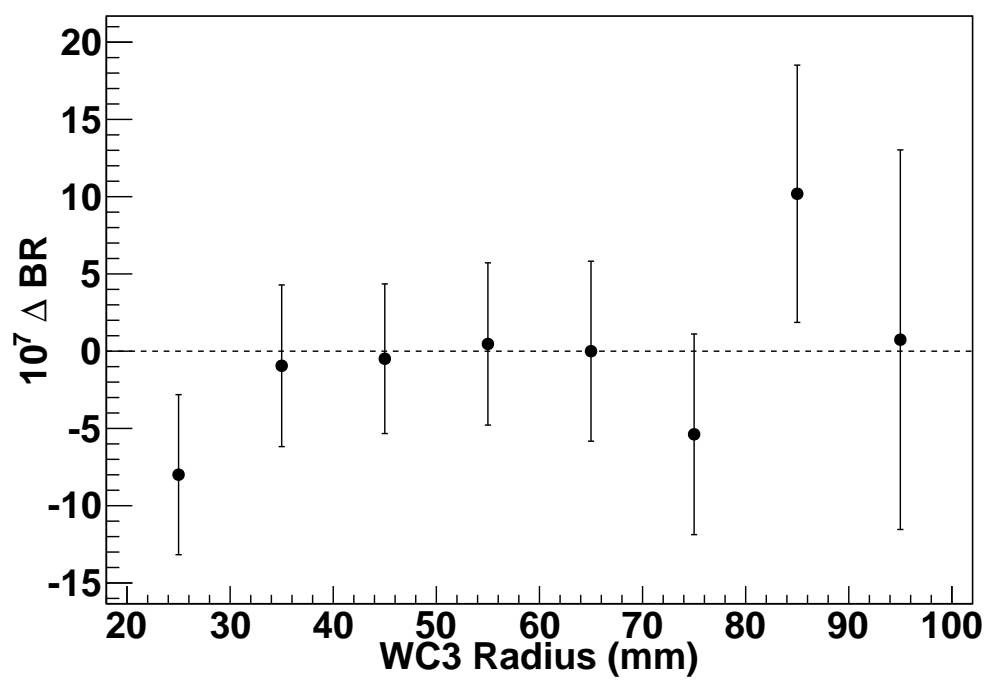

Figure 5: The variation in the branching ratio as a function of the distance between the reconstructed positron track and the centre of WC3. Each point represents the difference between the branching ratio using events in a $10 \mathrm{~mm}$ ring, from the branching ratio using the nominal radius $<60 \mathrm{~mm}$ cut. Both statistical and systematic uncertainties are included, but statistical uncertainty dominates; the error bars are therefore largely uncorrelated, since each point uses a statistically independent sample.

\section{Conclusions}

Once these checks had been passed and all known sources of systematic error had been evaluated, the blinding factor was removed. After all corrections, the result $R_{\pi}=(1.2344 \pm$ 0.0023 (stat) \pm 0.0019 (syst) $) \cdot 10^{-4}$ was obtained. The table of systematic uncertainties coming from the corrections is given in Table 2. The result confirms charged lepton universality at the $0.1 \%$ level.

Analysis of the full data set is underway; The 2011 data set is about 1.5 times larger than the 2010 data set discussed here, and the 2012 data set is about five times larger. A first version of the 2011 analysis has been completed and is in the process of being checked; thus, it is not yet unblinded. The result is $R_{\pi}=(1.2 x x x \pm 0.0018$ (stat) \pm 0.0013 (syst $\left.)\right) \cdot 10^{-4}$. The 2012 analysis is still underway; the statistical error is $0.09 \%$, but systematic effects still limit the precision. Combining all the data sets will result in a statistical error of $0.07 \%$. Efforts to reduce the systematic error from the low-energy tail, in particular, are ongoing; to achieve the goal of $0.1 \%$ total error it must be reduced to the $0.05 \%$ level.

\section{Acknowledgments}

This work was supported by the National Sciences and Engineering Research Council and TRIUMF through a contribution from the National Research Council of Canada, and by the Research Fund for the Doctoral Program of Higher Education of China, by CONACYT doctoral fel- 


\begin{tabular}{c|c|c|c|} 
Correction & Value & Statistical error & Systematic error \\
\hline Acceptance & 0.9991 & & 0.0003 \\
Low-energy tail & 1.0316 & & 0.0012 \\
Other & 1.0004 & & 0.0008 \\
\hline \hline$R_{\pi}^{\text {corr }}\left(10^{-4}\right)$ & 1.2344 & 0.0023 & 0.0019
\end{tabular}

Table 2: Systematic errors of the corrected branching ratio.

lowship from Mexico, and by JSPS KAKENHI Grant numbers 18540274, 213450059, 24224006 in Japan. We are grateful to Brookhaven National Laboratory for the loan of the crystals, and to the TRIUMF operations, detector, electronics, and DAQ groups for their engineering and technical support.

\section{References}

[1] A. Aguilar-Arevalo et al, Improved measurement of the $\pi \rightarrow e v$ branching ratio, Phys. Rev. Lett. 115, 071801 [hep-ex/1506.05845].

[2] M. Antonelli et al, Flavour physics in the quark sector, Phys. Rep. 494, 197.

[3] V. Cirigliano and I. Rosell, Two-Loop effective theory analysis of $\pi(K) \rightarrow e \bar{v}_{e}[\gamma]$ branching ratios, Phys. Rev. Lett. 99, 231801 [hep-ph/0707.3439]

[4] D.A. Bryman, W. Marciano, R. Tschirhart, T. Yamanaka, Rare kaon and pion decays: incisive probes for new physics beyond the standard model, Ann. Rev. Nucl. Part. Sci. 61, 331-354.

[5] D.I. Britton et al, Measurement of the $\pi \rightarrow e v$ branching ratio, Phys. Rev. D 49, 28.

[6] G. Czapek et al, Branching ratio for the rare pion decay into positron and neutrino, Phys. Rev. Lett. 70, 17.

[7] W. Loinaz, N. Okamura, S. Rayyan, T. Takeuchi, NuTev anomaly, lepton universality, and nonuniversal neutrino-gauge couplings, Phys. Rev. D 70, 113004 [hep-ph/0403306].

[8] A. Aguilar-Arevalo et al, Detector for measuring the $\pi \rightarrow e v$ branching fraction, Nucl. Instrum. Meth. A 791, 38 [hep-ex/1505.02737].

[9] A. Aguilar-Arevalo et al, High purity pion beam at TRIUMF, Nucl. Instrum. Meth. A 609, 102 [hep-ex/1001.3121]. 